\title{
Schulentwicklung im Spannungsfeld zwischen gesellschaftlichen und individuellen Anforderungen
}

\section{Gestaltungs- und Handlungsansätze für ein organisationales Gestaltungsmodell »Inklusive Schule»}

Fanny Hösel, Ariane Bößneck \& Anzhela Preissler

Journal für Psychologie, 27(2), 212-236

https://doi.org/10.30820/0942-2285-2019-2-212

www.journal-fuer-psychologie.de

\section{Zusammenfassung}

Lehrkräfte an deutschen Schulen werden mit der bildungspolitischen Forderung nach gleichberechtigter Teilhabe aller Schüler*innen nicht nur mit Blick auf die Gestaltung ihres Kerngeschäfts Unterricht vor enorme Herausforderungen gestellt. Inklusion rückt auch als organisationale und prozessuale Aufgabe der Schulentwicklung in den Verantwortungsbereich aller schulischen Akteure*innen. Noch immer mangelt es an wissenschaftlich begleiteten und aufeinander bezogenen Strukturen und Prozessen, die Bedarfe der Schulen analysieren, Unterstützungsmaßnahmen bestimmen und in konkrete Handlungen übersetzen. Ein vielversprechendes Gestaltungsmodell für Schulentwicklung, so die Erkenntnisse des laufenden Projektes »SING - Schule inklusiv gestalten. Entwicklung fachdidaktischer und organisatorischer Strukturen einer inklusiven Schule«, stellt die Balanced Scorecard (BSC) dar. Die aus der Unternehmungsführung stammende Managementmethode findet u. a. bereits im Non-Profit Bereich Anwendung. Für den schulischen Bereich wurde sie adaptiert und zu einer »strategischen Landkarte inklusiver Schulentwicklung « weiterentwickelt, um inklusive Schulentwicklungsprozesse systematisch zu unterstützen und zu gestalten. Der Beitrag stellt erste Einblicke in die laufende Projektarbeit bereit, indem eruierte Dimensionen inklusiver Handlungsfelder an Schulen skizziert werden. Diese werden im Rahmen eines groß angelegten Mixed-Method-Designs an fünf verschiedenen Pilotschulen wissenschaftlich evaluiert.

Schlüsselwörter: Schulentwicklung, Inklusion, Organisationsentwicklung, Prozessmanagement, strategische Landkarte 


\section{Summary}

School development between social and individual requirements. Design and action approaches for an organizational model »Inclusive school«

The educational policy requirement for equal participation presents enormous challenges for teachers at German schools not only with regard to the design of their core business. Inclusion also comes under the responsibility of all school actors as an organizational and procedural task of school development. There is still a lack of scientifically accompanied and interrelated structures and processes that analyze the needs of schools, determine support measures and translate them into concrete actions. A promising organizational model, according to the findings of the ongoing project »SING - School inclusive design. Development of didactic and organizational structures of an inclusive school «, is the Balanced Scorecard (BSC). The management method originating from the company management already applies in the nonprofit area. The aim is to adapt the BSC for the school sector and systematically support and design inclusive school development processes. The article provides initial insights into ongoing project work by outlining the dimensions of inclusive action fields in schools. These will be scientifically evaluated as part of a large-scale mixed-method design at five different pilot schools.

Key words: school development, inclusion, organizational development, process management, strategic map

\section{$1 \quad$ Einleitung}

Das zunächst bildungspolitisch motivierte Anliegen, das Thema Inklusion zu einem zentralen Aufgabenfeld forschungspraktischer Bemühungen zu erklären, hat mittlerweile seinen Niederschlag in konkreten Initiativen, wie dem vom Bundesministerium für Bildung und Forschung (BMBF) geförderten Verbundprojekt SING - Schule inklusiv gestalten ${ }^{1}$ gefunden. Eines der erklärten Ziele des Projekts - die Ausbildung fachdidaktischer Kompetenzen zur Gestaltung eines inklusiven Unterrichts des (künftigen) schulischen Personals ${ }^{2}$ - folgt dem Verständnis der notwendigen Einbettung jeglicher Professionalisierungsbemühungen in den organisationalen Kontext des Handlungsfeldes Schule. Ausgangspunkt bildet die Hypothese, dass Schulen mit Inklusionsbestrebungen vor der enormen Herausforderung stehen, gelingende Veränderungsprozesse auf sämtlichen Handlungsebenen und unter Einbezug aller beteiligter Akteure*innen zu erarbeiten, zu strukturieren und umzusetzen ${ }^{3}$. Obgleich das Kerngeschäft des Unterrichts als Ebene der direkten Interaktion das Zentrum von Inklusionsbemühungen im schulischen Kontext bildet, wird im vorliegenden Beitrag forschungsleitend davon ausgegangen, dass Inklusion über den je individuellen Verantwortungsbereich 
der einzelnen schulischen Akteure*innen hinaus als organisationale und prozessuale Aufgabe der Schulentwicklung zu denken und zu konzeptionieren ist. Dass dies eine besonders voraussetzungsreiche Aufgabe ist, zeigen nicht nur die fortfolgend präsentierten, ausgewählten Ergebnisse der im Projekt SING durchgeführten Befragungen aller beteiligten Akteure*innen (Eltern, Schüler*innen, schulisches Personal, Schulträger), sondern darüber hinaus deren Verwobenheit in gesellschaftliche wie auch individuelle Anforderungen. Die nachfolgenden Ausführungen gründen zu großen Teilen auf ausgewählten Ergebnissen des laufenden Teilprojekts »Organisationsentwicklung inklusiver Schulen «. Auf Basis qualitativer und quantitativer Erhebungen an fünf ausgewählten Pilotschulen in Sachsen konnten umfassende Erkenntnisse zu schulspezifischen Bedarfen und aktuellen Ressourcen, Strukturen und Prozessen sowie individuellen Einstellungen aller beteiligten Akteure*innen gewonnen werden.

Im Zentrum des vorliegenden Beitrags wird die Organisation Schule in ihrer strukturellen Eigenlogik fokussiert, um Handlungsfelder für die inklusionsbezogene Entwicklung von Schulen im Rahmen eines organisationalen Gestaltungsmodells »Inklusive Schule « zu beschreiben.

\section{Inklusionsbemühungen im Spannungsfeld vielfältiger Anforderungen}

Mit der Ratifizierung des Übereinkommens der Vereinten Nationen über die Rechte von Menschen mit Behinderung (UN-BRK) ist das deutsche Bildungssystem damit konfrontiert, inklusive Strukturen in den Schulen erfolgreich zu implementieren. Inklusion als Leitbild meint hier die gleichberechtigte Teilhabe an der Gesellschaft als Menschenrecht. Besonders relevant ist dabei der Artikel 24 - Bildung, indem die Vertragsstaaten das Recht auf Bildung von Menschen mit Behinderung anerkennen und sich verpflichten, ein integratives Bildungssystem auf allen Ebenen und lebenslanges Lernen zu gewährleisten (Beauftragte der Bundesregierung für die Belange von Menschen mit Behinderungen 2017, 21f.). Dabei steht unter anderem die deutsche Fassung in der scharfen Kritik, zentrale Begriffe wie »inclusive « (als »integrativ« übersetzt) fehlerhaft übersetzt zu haben, ohne Einwände der Fach- und Behindertenverbände - eine mögliche Entschärfung des emanzipatorischen Gehalts dieser Konvention - zu berücksichtigen (vgl. Frühauf 2008). Wocken (2011) merkt diesbezüglich an, dass der Begriff Inklusion mittlerweile auch in politischen Debatten als Selbstverständlichkeit Einzug gehalten hat, allerdings auch mit unerwünschten Nebenwirkungen. Ein zum Teil inflationärer Gebrauch des Inklusionsbegriffes läuft Gefahr, ein notwendiges gemeinsames Verständnis inklusiver Bildung zu unterlaufen, nämlich den gemeinsamen Unterricht » von behinderten und nicht behinderten Kindern 
in den Primar- und Sekundarstufen I und II (das heißt alle Schulformen einschließlich Gymnasium) «(DIM 2011, 11) und dabei sonderpädagogische Schwerpunkte kooperativ und integrativ in das reguläre Schulsystem einzubeziehen. Darüber hinaus verschleiert es notwendige Anforderungen auf Bundesebene einer systematischen Finanzierung, Angaben über erstrebenswerte Organisationsformen inklusiver Bildung oder auch Ressourcenzuweisungen.

Zur Umsetzung der UN-BRK gab es sowohl auf Länderebene Initiativen, Schulgesetze anzupassen und die Dimension Inklusion in der Lehrerbildung zu etablieren ${ }^{4}$, als auch regionale und überregionale Bemühungen, »Inklusion als Qualitätsdimension von Schule in den Schulinspektionen zu verankern « (Moser und Egger 2017, 9). Das dahinterliegende Bestreben richtet sich auf den sukzessiven Abbau von Sondereinrichtungen (bspw. Förderschulen) ${ }^{5}$, in denen Kinder mit speziellem Hilfebedarf betreut werden, zugunsten der Schaffung von Strukturen und Prozessen in Regelschulen, die allen Kindern gleichermaßen gerecht werden. Dabei liegt es in der Verantwortung der Einzelschule selbst, »Inklusion in der Unterrichts-, Personal- und Schul(kultur)entwicklung [zu] verankern und dies auch in den Schulprogrammen dar[zu]stellen « (ebd.)

Die normativen gesellschaftlichen und bildungspolitischen Anforderungen schlagen sich für die Schulen als Qualitätsmerkmale einer inklusiven Schulentwicklung nieder. Nun ist die Forderung der Sicherstellung angemessener Curricula, Lernsettings, Lehrstrategien sowie Ressourcen und Partner*innenschaften (Salamanca Erklärung 1994, vgl. hierzu auch die UN-BRK 2008; Deutsche UNESCO-Kommission 2014; Deutscher Gewerkschaftsbund 2015) als Konsequenz nachvollziehbar. Gleichzeitig birgt sie mit Blick auf schulische Praxis die Gefahr, ohne Konkretisierungen, Handlungsleitfäden und Indikatoren ins Leere zu laufen. Auch der Blick auf bereits empirisch gewonnene Qualitätsebenen, wie die Ermöglichung von Zugängen, Erfahrungen der Teilhabe und Unterstützung aller Akteursebenen (vgl. hierzu Hillenbrandt 2014), kann lediglich als Ausgangspunkt genutzt werden, um deren Umsetzungsbestrebungen mit konkreten potenziellen Gelingensbedingungen zu versehen. Egger (2017) resümiert mit Blick auf derzeitige Ergebnisse der allgemeinen Schulqualitätsforschung, dass sich Qualitätsdimensionen guter Schulen und guter inklusiver Schulen scheinbar nicht wesentlich voneinander unterscheiden und vermutet, dass so Ängste von Pädagogen*innen genommen werden sollen, sich der Inklusionsaufgabe zu stellen.

Welche komplexen Herausforderungen und enormen Hürden sich mit der Bewältigung eines inklusiven Schulalltages und der Gestaltung inklusiver Schulen ergeben und inwieweit normative bildungspolitische Forderungen an inklusive Schulentwicklung und schulische Alltagsrealitäten auseinandertriften, zeigt beispielsweise eine Meinungsumfrage des forsa-Instituts (2017). Von den 2050 befragten Lehrer*innen befürworten zwar ca. 50\% einen inklusiven Unterricht ${ }^{6}$, prangern allerdings die schlechten Rahmenbedingungen an. Insbesondere wird dabei auf die ungenügende materielle und 
finanzielle Ausstattung der Schulen verwiesen sowie auf eine unzureichende Ausbildung der Lehrkräfte. Enormen Unterstützungsbedarf sehen die Lehrkräfte bei der Inklusion von Kindern mit dem Förderschwerpunkt emotionale-soziale Entwicklung, Lernen und geistige Entwicklung. Es fehlen vor allem eine regelmäßige Doppelbesetzung (Sonderund Allgemeinpädagogen*innen), gute Schulungsangebote, Maßnahmen zur Bewältigung physischer und psychischer Belastungen, Ansprechpartner*innen vor Ort sowie eine adäquate räumliche Ausstattung.

Hinzu kommt, dass knapp 60\% (59,6\%) des befragten schulischen Personals ( $\mathrm{n}=133)$ im Projekt SING für sich selbst keine oder kaum sonderpädagogische Kenntnisse diagnostiziert. Dies stellt eine große Herausforderung dar, da es vor allem die positive Einstellung des schulischen Personals sowie deren Kompetenzen im Umgang mit Heterogenität sind, die als zentrale Gelingensbedingung für die gemeinsame Unterrichtung von Kindern mit und ohne sonderpädagogischem Förderbedarf gelten (Heinrich, Urban und Werning 2013).

Bereits an dieser Stelle deutet sich das Spannungsfeld aus Anforderungsgeflecht und Umsetzungsrealität an. So diagnostiziert beispielsweise Amrhein (2011) in ihrer Studie eine »Deformation integrativen Lernens « infolge unveränderter institutioneller Vorgaben, in denen die Lehrkräfte den gemeinsamen Unterricht auf das »eben Machbare « reduzieren und sich damit - entgegen des ursprünglichen Plans - Differenzierung und Separierung weiter verschärfen. Obgleich einzuschränken ist, dass dies sicherlich keine umfassende Diagnose spiegelt, sind potenzielle Gründe doch ernst und als Herausforderungen wahrzunehmen: fehlende Fachkonzepte, mangelnde Sensibilität und Qualifikation, ausbaufähige Rahmenbedingungen.

Zwei weitere Perspektiven auf Inklusion, die im Kontext entsprechender Schulentwicklungsbestrebungen zusätzlich in Rechnung gestellt werden, sind die der Schüler*innen selbst wie auch der betroffenen Elternschaft. So berichten Fegert und Schepker (2014) von Beobachtungen »emotional überforderte[r] Kinder, die [nach der Auflösung von Sonderschulen in Schleswig-Holstein] zunächst in ihrer Klasse recht wohl gelitten waren, aber über den Status eines »Maskottchens « nie hinauskamen « (298). Konsequenzen waren Einsamkeit, das tägliche Erleben von Unterlegenheit bis hin zur Exklusion aus schulinternen Gruppen. Zu einer ähnlichen Einschätzung kommt auch Schwab (2015), die den aktuellen Forschungsstand dahingehend zusammenfasst, dass »SchülerInnen mit SPF [sonderpädagogischem Förderbedarf] in Integrationsklassen einem sozialen Ausgrenzungsrisiko unterliegen. Sie haben weniger Interaktion mit Peers, weniger FreundInnen, werden häufiger zurückgewiesen und fühlen sich einsamer als ihre MitschülerInnen ohne Behinderung « (178). Auf der anderen Seite ist die Einstellung von Schüler*innen mit und ohne SPF gegenüber Peers mit Behinderungen (körperlich, geistig, Lernbehinderung, Verhaltensauffälligkeiten) »eher als neutral bis vorsichtig positiv einzustufen « (ebd., 183). 
Aufseiten der Eltern wirken sich insbesondere deren Einstellungen als »wesentliche Einflussgröße bei der Implementierung und Umsetzung integrativer ${ }^{7}$ schulischer Maßnahmen « aus (Trumpa et al. 2014, 243). Hess et al. $(2019,8)$ kommen zu dem Ergebnis, dass insgesamt 66\% der Eltern eine gemeinsame Unterrichtung von Kindern mit und ohne Beeinträchtigung befürworten. Hierbei differieren die Antworten zwischen denjenigen Eltern, deren Kinder eine Inklusionsschule besuchen zu denen, deren Kinder keine Inklusionsschule besuchen. Letztgenannte Gruppe spricht sich nur zu $61 \%$ für eine gemeinsame Unterrichtung aus, während bei erstgenannter Gruppe eine 78-prozentige Zustimmung herrscht.

Ergebnisse aus dem Projekt SING zeigen, dass knapp 50\% der befragten Eltern $(n=407)$ davon ausgehen, dass Kinder mit einem Förderbedarf im gemeinsamen Unterricht überfordert sind und gleichzeitig ist für 34,9\% vorstellbar, dass Kinder ohne Förderbedarf benachteiligt werden. Im Forschungsprojekt $>$ Einstellungsforschung zu Inklusion $(\mathrm{EFI})<$ wird weiter differenziert, »dass die Chancen des sozialen Lernens, des Abbaus von Vorurteilen und gegenseitigen Berührungsängsten positiver bewertet werden, als die Gelegenheiten zur individuellen Förderung und Unterstützung von Lernen und Schulleistungen in gemeinsamen Lernsettings « (Trumpa et al. 2014, 251). Erstaunlich ist, dass es vor allem die Eltern mit Erfahrungen im Inklusionskontext sind, die eine Leistungsförderung im Zuge inklusiver Unterrichtung kritischer sehen als Eltern ohne Inklusionserfahrung - zu dieser Einschätzung kommt eine jüngst veröffentlichte Studie des infas Instituts für Sozialwissenschaften (Hess et al. 2019, 13).

Zusammenfassend lässt sich sagen, dass gesellschaftliche und bildungspolitische Anforderungen wie auch akteursspezifische Erwartungen und Einstellungen im Rahmen von Inklusion Schulen vor enorme Herausforderungen stellen. Dies betrifft sowohl das Kerngeschäft des Unterrichts (Professionalisierung der Lehrkräfte, Gestaltung von Lernsettings) als auch die Personalentwicklung, der Einbezug aller am Schulprozess beteiligten Akteur*innen und die Schulorganisation. Die Bewältigung des schulischen Alltags bedarf der Berücksichtigung situativer und personaler Gegebenheiten der jeweiligen Einzelschule, damit Inklusion als Menschenrecht erfahrbar wird und nicht an seiner pädagogischen Ideologie scheitert.

\section{Inklusive Schulentwicklung als Organisationsentwicklung}

Eine intensive Auseinandersetzung mit Schulentwicklung erscheint im schulischen Alltag meist nicht vordergründig, ist doch das Kerngeschäft von Pädagogen*innen der Unterricht an sich. Dabei wird häufig nicht bedacht, dass die Organisation Schule und deren Entwicklung einen unmittelbaren Einfluss genau auf dieses Kerngeschäft haben, indem sie (1) den sozialen Rahmen für das Handeln von Pädagogen*innen bil- 
det (bspw. Umgang mit Konflikten), (2) einen verbindenden Rahmen zwischen dem Handeln der Lehrerkräfte und Schüler*innen sowie den gesamtgesellschaftlichen Anforderungen darstellt (bspw. Curriculare, Heterogenität der Schülerschaft) und (3) die Organisation von Lehr- und Lernprozessen mitbestimmt (bspw. die Ausweitung des Bildungssystems) (Langenohl 2008). Schulische Organisationen liegen als Mesosystem an der Schnittstelle, wenn es um Veränderungen, sich ergebender Herausforderungen und Neuerungen sowohl auf Makroebene (Gesellschaft, überregionale und regionale Bildungsstrukturen) als auch auf Mikroebene (Lernsettings, individuelle* ${ }^{*}$ Schüler*in) geht.

Schulentwicklung nimmt dabei das gesamte System Einzelschule in den Blick und bezieht sich auf die sich wechselseitig beeinflussenden Bereiche der Organisation, des Unterrichts und des Personals (Rolff 2016). Konkret wird damit die systematische Entwicklung der Einzelschule auf der Team-, Abteilungs- oder Schulebene durch das schulische Personal selbst gemeint; und zwar als Entwicklung der Bedingungen des täglichen Unterrichts sowie als gemeinsame curriculare und methodische Analyse (Wilbers 2012, 400). Die Synthese aller drei Dimensionen im Rahmen des Inklusionskontexts, so Amrhein (2014), umfasst die Schaffung inklusiver Strukturen, Unterrichtsentwicklung als Entwicklung Inklusiver Pädagogik und Personalentwicklung als Professionalisierung für Inklusion.

Trotz existenter Qualitätskriterien für die Gestaltung inklusiver Schulentwicklung in Deutschland mangelt es nach wie vor an wissenschaftlich begleiteten und aufeinander bezogenen Strukturen und Prozessen, die es ermöglichen, Entwicklungsbedarfe der Einzelschule zu analysieren, deren notwendige Unterstützungsmaßnahmen zu bestimmen und in konkrete Handlungen zu übersetzen. Darüber hinaus werden Schulentwicklungsaktivitäten häufig als zusätzliche Belastungen neben dem schulischen Alltagsgeschäft wahrgenommen und die verantwortlichen Akteure*innen fühlen sich in der Umsetzung entsprechender Maßnahmen häufig alleingelassen. Wagner und Rückmann (2017) stellen heraus, dass für gelingende Schulentwicklungsmaßnahmen und Evaluationen für die Lehrkräfte ein unmittelbarer Nutzen deutlich gemacht werden muss (bspw. spürbare Veränderungen im unterrichtlichen Handeln durch Schulentwicklungsmaßnahmen). Unbedingte Voraussetzung, bereits im Prozess der Initiation und Konzeption, ist demzufolge die aktive Einbeziehung der am späteren Umsetzungsprozess Beteiligten, insofern sich die Schulen als »sich selbst gestaltende Handlungseinheiten « verstehen wollen und nicht als »zellulärer Haufen oder fremdbestimmte Dienststelle « (Berkemeyer 2010, 39). Zudem muss für die Unterstützung der Implementierung konkreter Ziele und Prozesse ein Prozessmanagement etabliert werden, was gemeinsam mit den Schulen zu entwickelnde Prozesse plant, steuert und evaluiert.

Ausgangspunkt für die empirische Bearbeitung bildeten im Projekt SING dabei zunächst die Fragen, welche Schulentwicklungsprozesse in den untersuchten Schulen 
bereits existent sind (Analyse des IST-Standes), wie sie sich bisher gestalten (bspw. Verantwortlichkeiten, Prozesse, Steuerung, Partizipation, Kommunikation) und welche Formen der Dokumentationen von Schulentwicklungsprozessen vorhanden sind (auch mit Bezug auf schulinterne Evaluation). Darüber hinaus lag ein besonderes Augenmerk auf der Analyse von Ressourcen, Bedarfen und schulformspezifischen Herausforderungen für inklusive Schulentwicklungsprozesse (Bedarfsanalyse).

Ziel der Forschungsbemühungen insgesamt ist es, ein anwendungspraktisches Gestaltungsmodell für inklusive Schulentwicklungsprozesse zu entwickeln. Dieses Modell fußt auf empirisch gesicherten Daten, die innerschulische Planungs-, Reflexions- und Entwicklungsprozesse systematisch sichtbar macht, strukturiert und dabei alle am Prozess beteiligten Handlungsebenen systematisch aufeinander bezieht. Um differenten organisatorischen, strukturellen und bildungsbezogenen Anforderungen unterschiedlicher Schulformen gerecht zu werden, wie auch den Blick auf Transitionen innerhalb einer Schüler*innenbiografie und damit verbundenen Herausforderungen für alle beteiligten Akteure*innen zu schärfen, wurden sowohl eine Grundschule, eine Oberschule, eine integrierte Grund- und Oberschule, ein Gymnasium sowie eine Berufsschule in das Sample einbezogen. Eine der forschungsleitenden Maßgaben war es, die Perspektiven aller am schulischen Geschehen beteiligten Personen zu berücksichtigen. Entsprechend wurden zum einen die Sichtweisen der am Schulentwicklungsprozess beteiligten Fachexperten analysiert (Experten*inneninterviews mit den Schulleitungen und Schulträgern, Gruppendiskussionen mit ausgewählten Lehrer*innengruppen, quantitative Fragebogenerhebung des gesamten Kollegiums). Zum anderen konnten mithilfe quantitativer Fragebögen Perspektiven der Schüler*innen sowie deren Eltern erhoben werden. Lokale Gegebenheiten der Schule (bspw. Standort der Schule, Anteil der Schüler*innen mit Migrationshintergrund; Einkommen der Eltern) wurden ebenfalls einbezogen, um schulformspezifische Rahmenbedingungen festzuhalten und diese im Entwicklungsprozess zu berücksichtigen. Darüber hinaus diente eine deskriptive Homepageanalyse der jeweiligen Schulen dazu, sowohl Formen der Außendarstellung der Schule als auch Möglichkeiten der digitalen Vernetzung schulischer Akteure*innen zu erfassen und diese im Rahmen der Projektlaufzeit begleitend weiterzuentwickeln.

\subsection{Theoretische Vorüberlegungen zu einem organisationalen Gestaltungsmodell für inklusive Schulen}

Ein vielversprechendes Instrument, was auch für Schulentwicklung nutzbar gemacht werden kann, stellt die Balanced Scorecard (BSC) dar. Anfang der 1990er Jahre von Kaplan und Norton entwickelt, ist die BSC eine aus der Unternehmungsführung stammende Managementmethode, welche bereits in vielfältiger Form Anwendung in 
zahlreichen Institutionen der Wirtschaft, aber auch im Non-Profit Bereich und Einrichtungen des Bildungswesens (Universitäten, Schulen) findet. Der Begriff Balanced verweist auf eine Ausgeglichenheit verschiedener Dimensionen und Perspektiven, die für die Erreichung einer gemeinsamen Vision inhaltsfüllend sind. Darüber hinaus soll auch die Ausgewogenheit hinsichtlich der Art und Terminierung der Ziele Berücksichtigung finden, indem »kurzfristige und langfristige, von den Beteiligten gemeinsam vereinbarte Ziele Gegenstand einer BSC werden. Die Orientierung an einem solchen »strategischen Steuerungsinstrument « birgt den Vorteil, »dass Zielvorgabe und Zielerreichung in einem größeren strategischen Zusammenhang entworfen werden « (Dubs 2006, 136). Entsprechend werden - abgeleitet aus einer gemeinsamen Vision - für jede Perspektive:

1. Ziele formuliert,

2. für jedes Ziel werden Messgrößen festgelegt (bringen Ziele auf den Punkt, diagnostischer und strategischer Natur),

3. es müssen Maßnahmen formuliert, die zur Zielerreichung führen und

4. erforderliche Ressourcen analysiert werden.

Für das vorliegende Projekt wurden die maßgeblichen Logiken dieser Methode sowie das Grundschema so adaptiert, dass sie für inklusive Schulentwicklungsprozesse nutzbar gemacht werden konnten, ohne die besondere Eigenlogik der Organisation Schule aus den Augen zu verlieren. Konkret erweiterte sich beispielsweise die strategische Zuweisung von (aufeinander abzustimmenden) Zielen zu ursprünglich vier Gruppen (Dubs 2006 für den schulischen Kontext: Anspruchsgruppen; Management-, Leistungserbringungs- und Unterstützungs-prozesse; Schulentwicklung; Finanzen) auf nunmehr fünf sogenannte Handlungsdimensionen, die zudem einer anderen als hier skizzierten Differenzierungslogik folgen (siehe nächstes Abschnitt). Alle fünf Perspektiven, für die - abgeleitet aus der gemeinsamen Vision - konkrete Ziele, Maßnahmen, Messgrößen und Ressourcen formuliert wurden, konnten außerdem um Akteursebenen ergänzt werden. Das heißt, für das zentrale Ziel inklusiver Schulentwicklung erfolgten im Sinne eines zielgerichteten Prozessmanagements dimensionsspezifische Handlungs-implikationen für die Schulleitung, das schulische Personal, die Schüler*innen und auch deren Eltern.

Im Kontext des Projekts versteht sich die Balanced Scorecard als Managementinstrument für die zielgerichtete Ingangsetzung und Durchführung von Schulentwicklungsprozessen. Anders als bei Dubs (2006), in dem Schulentwicklung » nur « innerhalb einer Perspektive behandelt wird, ist sie im fortfolgend skizzierten Modell das rahmende Element. Sämtliche, den einzelnen fünf Dimensionen zugewiesene Ziele, Maßnahmen und Akteure*innen sind als Teilelemente eines ganzheitlichen, auf Inklusion gerichteten Schulentwicklungsprozess zu verstehen. 
Gemäß dem eingangs aufgeführten Interesse an organisationsbezogenen Bedingungen, liegt der Schwerpunkt der folgenden Ausführungen auf dem Bereich der Organisationsentwicklung als zentrales Arbeitsfeld der Schulentwicklung ${ }^{8}$. Schule versteht sich in diesem Blickwinkel als Lernende Organisation (Rolff2007), die Ziele und Qualitätskriterien einer inklusiven Schule definiert, Teilhabe ermöglicht und den an Schulentwicklung beteiligten Akteuren entsprechende Verantwortungen zuweist. Gleichzeitig kommt das Umfeld der Schule als Schule-Umwelt-Beziehung in den Blick, um »sich für Eltern zu öffnen, zu Teilen regionaler inklusiver Bildungslandschaften zu werden und Kooperations- und Unterstützungssysteme aufzubauen « (Görtler 2017, 146).

Die anschließenden Ausführungen zur »strategischen Landkarte inklusiver Schulentwicklung $\ll{ }^{9}$ spiegeln den derzeitigen Arbeitsstand des Teilprojektes wieder [erste Jahreshälfte 2019]. Das skizzierte Modell beruht dabei sowohl auf der Basis praxiserprobter Ansätze ${ }^{10}$ in der Arbeit mit der Balanced Scorecard im schulischen Bereich sowie umfassender theoretischer und empirischer Analysen zu inklusiven Schulentwicklungsprozessen. Zentralen Stellenwert für die inhaltliche Benennung relevanter Prozesse und Strukturen der einzelnen Handlungsfelder nahm unter anderem der bereits seit einigen Jahren erfolgreich erprobte Index für Inklusion (2003) ein. Dabei konnte der im Index angelegten Strukturierungslogik in Dimensionen (Handlungsfelder) und zugeordneten Indikatoren (Messgrößen) für das eigene Modell gefolgt werden. Einer der zentralen Unterschiede allerdings liegt in der Aufgliederung der Dimensionen. Während der Index in »inklusive Kultur «, »inklusive Strategie « und »inklusive Praxis « unterteilt, folgt das Gestaltungsmodell inklusive Schule einer Unterscheidung von fünf verschiedenen Handlungsbereichen mit je konkreten Aufgaben für differenzierte Akteursgruppen. Zwar spielen gemeinschaftsbildende Prozesse, inklusive Werte und Strukturen, Unterstützungs-modalitäten und inklusive Praktiken eine Rolle (Boban und Hinz 2003), werden aber unter Ergänzung weiterer Aspekte konkreten Verantwortlichkeiten zugewiesen, strukturell miteinander in Beziehung gesetzt und insgesamt in einen ganzheitlichen Managementprozess (als Schulentwicklung) integriert.

Das zunächst theoretisch entwickelte Modell als Ergebnis einer theoriebasierten Exploration (Bortz und Döring 2000) wird in einem aktuell laufenden Schritt um induktiv gewonnene Kategorien aus der qualitativen Inhaltsanalyse (Mayring 2008) der erhobenen Daten ergänzt. Auf dieser zunächst modellhaften Universal-Vorlage war es dann in einem zweiten Schritt möglich, den Status quo der Schulen, bezogen auf ihre aktuellen Initiativen zu Inklusion, abzubilden und als Ausgangspunkt für schulspezifische Entwicklungsbemühungen zu nutzen. Die dafür notwendigen Erkenntnisse zur schulspezifischen Befüllung der einzelnen Dimensionen konnten im Zuge der bereits beschriebenen Datenerhebung und Aufbereitung gewonnen werden.

Im Rahmen eines gemeinsamen Workshops mit Schulleitung, Lehrkräften sowie interessierten Eltern werden darüber hinaus die Ergebnisse gemeinsam diskutiert und 
halten gemäß ihrer eingeschätzten Priorisierung Einzug in das schulspezifische Gestaltungsmodell. Erst daran anschließend folgt die Formulierung schulspezifisch relevanter Prozesse und Maßnahmen entsprechend den von den Schulen selbst formulierten Zielen im Kontext inklusiver Schulentwicklungsbestrebungen.

\subsection{Strategische Landkarte inklusiver Schulentwicklung - ein Gestaltungsmodell}

Im Zentrum des entwickelten Gestaltungsmodells (Abbildung 1), das hier in Anlehnung an die obigen Ausführungen als strategische Landkarte inklusiver Schulentwicklung bezeichnet wird, steht - wie es auch die BSC verlangt - die jeweilige Vision der Schule. Sie beschreibt einen erwünschten, zukünftigen Zustand und gibt die Richtung an, in die sich die Organisation entwickeln soll als »konkretes Zukunftsbild, nahe genug, dass wir die Realisierbarkeit noch sehen können, aber schon fern genug, um die Begeisterung der Organisation für eine neue Wirklichkeit zu wecken « (The Boston Consulting Group 1988). Je nachdem, wie die Schulen für sich diese Vision einer inklusiven Schule ausdeuten ${ }^{11}$, schlägt sich dies in der inhaltlichen Beschreibung des jeweiligen Leitbildes nieder, in dem konkrete Entwicklungsziele als Sollzustände, das Selbstverständnis der Schulgemeinde sowie Wertvorstellungen zum Ausdruck kommen (Egger 2017, 40). Zwar wird von einem gemeinsam getragenen Werteverständnis bezüglich Inklusion ausgegangen, im Sinne eines gleichberechtigten Zugangs und einer die Entwicklung des Einzelnen unterstützende Teilhabe am Unterricht und dem Schulleben (Moser und Egger 2017, 24). Bewusst wird aber davon Abstand genommen, den Schulen eine einheitliche Begriffsdefinition von Inklusion vorzulegen. Steuerungsstrategisch würde dies einen Top-Down Prozess implizieren, der von Extern gesetzt wird. Zentrales Anliegen ist es dem gegenüber, »dass vielfältige Interpretationen in einem machtvollen, interessengeleiteten Raum zu Reformulierungen [oder auch] lokalen Interpretationen gelangen « (Moser 2017, 16), um den je schulspezifischen Rahmenbedingungen und Bedarfen zu entsprechen.

Die formulierten Entwicklungsziele des Leitbildes avancieren zur handlungsleitenden Maßgabe für die Befüllung im Sinne der gemeinsamen Bearbeitung der fünf verschiedenen Handlungs- und Steuerungsdimensionen: Pädagogische Professionalisierung, Schulumwelt, Unterricht, Schulleben sowie Digitalisierung.

Zur konstruktiven, zunächst analytischen und folgend praktischen Bearbeitung, ist jede der Dimensionen in die Ebenen des Inhaltsmanagements sowie des Prozessmanagements unterteilt, die dann in der Umsetzung zusammenspielen. Sie spiegeln zum einen die für die Schule relevanten Themen wieder (Inhalt) und werden mit Maßnahmen zu ihrer Bearbeitung unterlegt (Prozess): »Prozessmanagement für Schulen wird hier als 


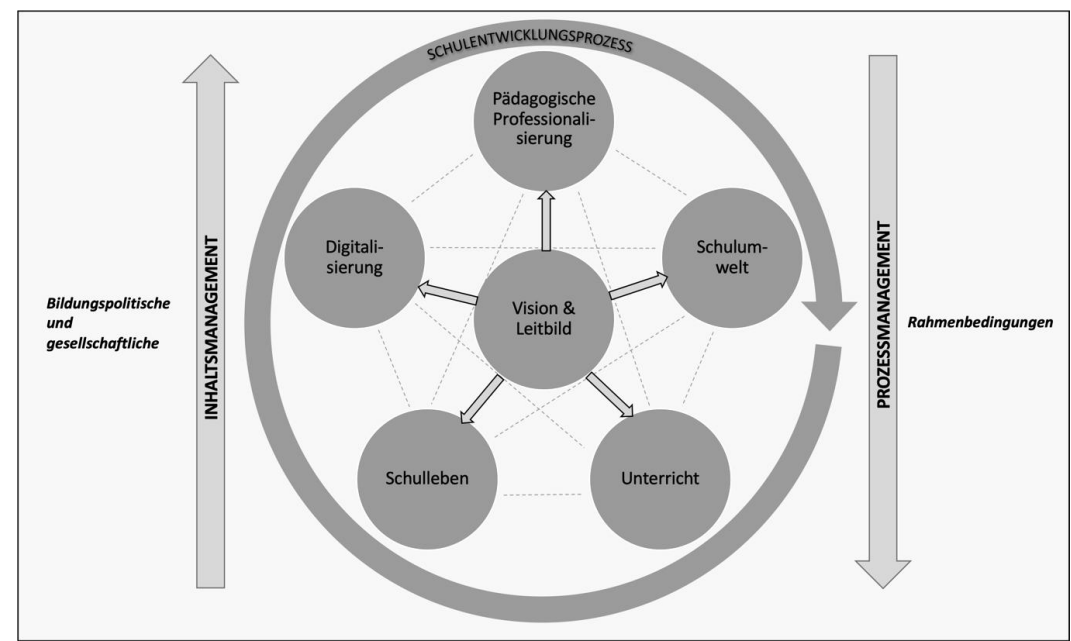

Abbildung 1: Strategische Landkarte inklusiver Schulentwicklung (Stand 07/2019) ${ }^{12}$

ein umfassendes System der Schule zur Identifizierung, Modellierung, Überwachung und Optimierung von (Schul-)Prozessen verstanden« (Berglehner und Wilbers 2015, 33). Die Schwerpunktsetzung schulspezifischer Entwicklungs-Anliegen in den einzelnen Dimensionen folgt gemäß BSC den Fragen:

a) auf Inhaltsebene (Was wird entwickelt?):

$>\quad$ Welche Themen sind für uns aus der vorab formulierten Vision heraus in diesem Feld relevant?

$>\quad$ Welche Ziele lassen sie konkret für jede Handlungsdimension festlegen und

> Wodurch können wir sie im Anschluss überprüfen (Qualitätskriterien als Messgrößen und Zielwerte)?

b) auf Prozessebene (Wie wird entwickelt?):

$>\quad$ Mit welchen Maßnahmen können die Ziele erreicht werden?

$>\quad$ Wie kann der Erfolg überprüft werden (Evaluation)?

Die Zuweisung von Verantwortlichkeiten erhält im Modell eine eigene, strategisch zu bearbeitende Ebene: die Akteursebene (Wer entwickelt?). Grund hierfür liegt in der Ambition, den Einbezug aller beteiligten Akteure*innen - so auch Eltern und Schüler*innen, neben dem schulischen Personal und der Schulleitung - gleichermaßen sicherzustellen. Inklusion wird als Aufgabe verstanden, »die alle Akteure[*innen] einer Schule betrifft « (Urton et al. 2018, 40), daher sollte markiert werden, wer für die Durchführung einzelner Prozessschritte verantwortlich und zuständig ist (Berglehner 
und Wilbers 2015, 20). Entsprechend wird auf dieser Ebene die Frage danach gestellt, wer sich für die Umsetzung der Maßnahmen verantwortlich zeichnet.

Die Ressourcen (Womit wird entwickelt?) umfassen im vorliegenden Modell die zur Verfügung stehende personelle, materielle und räumliche sowie finanzielle Ausstattung einer Schule. Sie bilden gleichzeitig die Grundlage für sämtliche Bestrebungen zur Gestaltung eines inklusiven Schullebens, sind andererseits aber auch Grenzmarkierer. Ihr (Nicht-)Vorhandensein sowie deren Steuerung beeinflussen maßgeblich die Umsetzung von Inklusion (Melzer und Hillenbrand 2015). In Hinblick auf die personellen Ressourcen richtet sich der Bedarf auf speziell geschultes Personal, wie Sonderpädagogen*innen, Sozialarbeitende, Psychologen*innen oder Inklusionsassistenten*innen, der in allen Schulen als verbesserungswürdig angesehen wird. Knapp 50\% der Eltern und 32,1\% des Lehrpersonals bewerten die personelle Ausstattung an den untersuchten Schulen als mangelhaft bis ungenügend. Bedarf besteht an den Schulen darüber hinaus vor allem an zeitlichen Ressourcen - im Unterrichtskontext beispielsweise » für die individuelle Förderung und die Zusammenarbeit im multiprofessionellen Team « (Santoli et al. 2008). Aber auch außerhalb des Kerngeschäfts werden zeitliche Verfügbarkeiten gleichermaßen als zwar zwingend notwendig, in der Realität jedoch als nicht ausreichend vorhanden markiert. Ein Mangel an ausreichenden Zeitfenstern ist die Basis für entstehende Folgeprobleme, die sich zum Beispiel in unstrukturierten Kommunikationsprozessen niederschlagen können.

Die räumlichen Ressourcen im Sinne zur Verfügung stehender Ausstattungen sowie materielle Ressourcen sind weitere Einflussfaktoren auf ein inklusives Schulklima (Artiles und Dyson 2005). Zwar, das zeigen bereits erste Ergebnisse der laufenden Studie, verfügt ein Großteil der Pilotschulen über entsprechende materielle Ressourcen und ausreichende Räumlichkeiten (Beratungsräume, Aufenthaltsräume etc.), eine barrierefreie Architektur (Aufzüge, behindertengerechte Toiletten etc.) und eine moderne Ausstattung (interaktive Tafeln, PC-Kabinette etc., organisationsinterne digitale Kommunikations- und Organisationsplattformen), insbesondere in Bezug auf die Nutzung letztgenannten Punktes werden allerdings neue Herausforderungen offenbar (siehe Dimension $>$ Digitalisierung $<$ ).

Die Dimension der pädagogischen Professionalisierung entspricht im Wesentlichen dem Anliegen der Personalentwicklung und stellt das gesamte Wissen, die Fähigkeiten wie auch die Motivation und Einstellung der an Inklusion beteiligten Akteure*innen, insbesondere des schulischen Personals in den Mittelpunkt. Für deren Förderung und zielgerichtete Entwicklung im Sinne einer Professionalisierung kommt der Schulleitung eine zentrale Rolle zu (Scheer und Laubenstein 2018). Neben Managementkompetenzen, die auf dieser Ebene vorhanden sein sollten, um im Sinne eines effizienten Schulmanagements einen strukturellen Rahmen für inklusive Schulentwicklung zu schaffen (hierzu gehören unter anderem die Akquise des Personals, die zielgerichtete 
Planung und Steuerung der für sonderpädagogische Förderung vorhandenen Ressourcen, das Treffen datengestützter Entscheidungen, der Aufbau einer für alle transparenten Informations- und Kommunikationsstruktur), ist es vor allem die professionelle Personalentwicklung, die in das Aufgabenspektrum der Schulleitung gehört. Für das potenzielle Ziel des Aufbaus des Kollegiums als Learning Community beispielsweise könnten entsprechende Messgrößen die Delegation von Entscheidungsprozessen sowie die Zuweisung von Handlungsspielräumen, regelmäßige Hospitationen und Kooperationen, das Erkennen und zielgerichtete Weiterentwickeln der Kompetenzen des schulischen Personals sein. Gleichermaßen liegt es andererseits in der Eigenverantwortung der angesprochenen Akteure*innen, die Bereitschaft zur aktiven Teilhabe zu signalisieren und dafür notwendige Kompetenzen weiterzuentwickeln. Die Befragung im Projekt SING zeigt, dass über $80 \%$ des befragten schulischen Personals motiviert ist, sich an (inklusiven) Schulentwicklungsprozessen zu beteiligen. Bezüglich der dafür notwendigen Kompetenzen wird im vorliegenden Modell für die Akteursebene der Lehrenden zwischen persönlichen Kompetenzen (Selbstwirksamkeit, Rollenbewusstsein, Wert-orientierung, Reflexionskompetenz), Sozial- und organisationalen Kompetenzen (wichtig hier vor allem die Nutzung von Handlungs- und Entscheidungsspielräumen aus eigener Initiative) sowie adaptive Lehrkompetenzen (Sach- und Fachkompetenzen, diagnostische, didaktische und Klassenführungskompetenzen) unterschieden. Im Kontext inklusionsorientierter Bildung meint letztgenannte die Fähigkeit einer Lehrperson, den »Unterricht so auf die individuellen Voraussetzungen und Möglichkeiten der Lernenden anzupassen, dass möglichst günstige Bedingungen für individuell verstehendes Lernen entstehen und beim Lernen aufrecht erhalten bleiben « (Becker et al. 2008, zit. nach Fischer et al. 2014). Die hierfür notwendige sonderpädagogische Kompetenz wird in den untersuchten Fällen der sächsischen Schulen vom befragten Lehrpersonal selbst als überwiegend unzureichend eingeschätzt: Knapp 60\% des Lehrpersonals gibt an, keine bis wenig ausgeprägte sonderpädagogische Kenntnisse zu haben. Offensichtlich werden an dieser Stelle umfassende(re) Qualifizierungsnotwendigkeiten.

Neben diesen verweisen die Ergebnisse der vorliegenden Untersuchung auf ein vergleichsweise hohes Belastungserleben des Lehrpersonals. Arbeitszufriedenheit definiert sich im vorliegenden Modell als » positiver emotionaler Zustand, der sich aus der Bewertung der eigenen Arbeit sowie der Arbeitserlebnisse ergibt « (Locke 1976, 1299). Eine hohe Ausprägung der Arbeitszufriedenheit und ein gleichermaßen geringes Belastungserleben wirken sich positiv auf eine gelingende inklusive Schulentwicklung aus (Urton et al. 2018). Mit Blick auf innerschulische Realitäten der Alltaxpraxen, die sich auch in den vorliegenden Daten spiegeln ${ }^{13}$, wird klar, dass gerade letztgenannter Punkt verstärkter Aufmerksamkeit bedarf und nicht einer ausschließlich ergebnisorientierten (Weiter-)Qualifizierungspraxis nachstehen darf. 
Da es zum Schluss immer die am Schulentwicklungsprozess beteiligten Akteure*innen sind, die die avisierten Maßnahmen zur Veränderung der Organisation mittragen und umsetzen müssen, unterliegen sämtliche Aktivitäten deren jeweils vorherrschender Haltung gegenüber Inklusion ${ }^{14}$.

Nach aktuellen Erkenntnissen sind eigene Überzeugungen mit Blick auf inklusive Schulentwicklungen eine Grundvoraussetzung und gleichzeitig zentraler Gelingensfaktor dafür, dass eine Transformation des Schulsystems hin zur Inklusion gelingen kann (Hennemann et al. 2018, 10; Urton et al. 2018, 42). In Abhängigkeit davon, wie Inklusion auch auf je individueller Ebene definiert und bewertet, welcher Stellenwert ihr an der Schule beigemessen, wie viel Sinnhaftigkeit ihr zugesprochen wird, und nicht zuletzt, welche emotionalen Erfahrungen mit der Thematik verbunden sind (Jordan et al. 1993), werden sich Schul-entwicklungsprozesse mehr oder weniger erfolgreich umsetzen lassen. Im Sinne inklusiver Schulentwicklungsbestrebungen bedeutet dies auch, »Meinungen bezüglich Vielfalt zu fördern « (Urton et al. 2018, 45). In Konsequenz impliziert das auch die Zulässigkeit unterschiedlicher Überzeugungen beispielsweise des Stellenwertes von Inklusion an der Schule. An dieser Stelle sind nicht nur das schulische Personal, sondern auch Schüler*innen wie auch die Eltern von Kindern mit und ohne Förderbedarf angesprochen (siehe auch Schulleben). Deren Einstellungen stellen sich als »entscheidend für das Gelingen von Inklusion « (de Boer et al. 2012; Hennemann et al. 2018, 9f.) dar. Nur in gegenseitigen Aushandlungsprozessen aller beteiligten Akteure*innen kann es zu gemeinsam geteilten Visionen kommen und Veränderungsprozesse angeregt werden (Rolff 2007, 14; Hennemann et al. 2018, 10).

Die Unterrichtsentwicklung umfasst hier primär die im Unterricht relevanten Aspekte der Didaktik/Methodik, der Unterrichts- und Sozialformen, der eingesetzten Medien und Materialen sowie der Heterogenität der Schüler*innenschaft. Damit rückt die Akteursebene des schulischen Personals in den Vordergrund wie auch die der Schüler*innenschaft als Rezipienten unterrichtlicher Maßnahmen. Da die wichtigsten Erziehungsund Bildungsprozesse im Unterricht stattfinden, gilt es, das Augenmerk auf diejenigen Gestaltungsaspekte förderlicher Lehr- und Lernformate zu richten, die Inklusion ermöglichen. Dazu zählen unter anderem die gezielte Beförderung positiver Gemeinschaftserfahrungen, die Schaffung eines Klimas der Akzeptanz, die Unterstützung sozialemotionaler Lernprozesse, sowie die Etablierung einer entwicklungs-bezogenen Feedbackkultur (Soodak 2003). Um den unterschiedlichen Bedarfen und Leistungsständen aller Schüler*innen gerecht zu werden, haben sich hochgradig differenzierte Lehr-, Lernmaterialien wie auch »differenzierte Unterrichtsmedien «(Bosse 2017,2018) als zentral für inklusiven Unterricht erwiesen. Sämtliche didaktische und methodische Bestrebungen einer differenzierten Unterrichtsgestaltung fußen letztlich auf der Anerkennung der Heterogenität und Vielfalt der Schüler*innenschaft, was eine Abkehr von Konzeptionen homogener Lerngruppen impliziert (Gomolla und Radtke 2009). Für die Lehrenden hat 
dies in Konsequenz die »Abstimmung von allgemeiner Förderung in der Klassengruppe und diagnostisch fundierter, individueller und intensiver Förderung bei Lernschwierigkeiten« (Wember 2013, 380) zur Folge (siehe auch pädagogische Professionalisierung).

Das Verbundprojekt SING ermöglicht dabei gewonnene Daten aller Teilprojekte (fachdidaktische Kompetenzen und organisatorische Strukturen) fortlaufend zusammenzuführen und zu ergänzen, wodurch der gesetzte Anspruch des Projektes im Sinne einer ganzheitlichen Konzeption inklusiver Schulentwicklung sowohl theoretisch als auch empirisch fundiert gerecht wird.

Die Schulumwelt als drittes Handlungsfeld umfasst externe Unterstützungen und Kooperationen, die für inklusive Entwicklungsbestrebungen genutzt werden und ressourcensichernde Funktionen ausüben (können) (Rolff 2007, 13). Neben Schulleitung und Lehrer*innenschaft sind in dieser Dimension auch wieder Perspektiven der Eltern angesprochen, die durch Einbindung in Netzwerk- oder Förderstrukturen einen zentralen Beitrag zur inklusiven Schulentwicklung leisten können. Für die untersuchten Fälle im Projekt SING liegt gerade hierin großes Handlungspotenzial: Knapp 50\% der befragten Eltern sind wenig bis gar nicht motiviert, sich an Schulentwicklungsprozessen zu beteiligen. Dabei können außerschulische Partner*innenschaften auf vielerlei Ebenen genutzt werden, sei es in Form synergetisch genutzter Zusammenarbeit (z. B. mit Betrieben), in Form ehrenamtlicher Unterstützung, beispielsweise durch die Übernahme außerschulischer Angebote, finanzieller Unterstützung oder auch wissenschaftlicher Begleitungen. Insbesondere für Maßnahmen der Übergangsgestaltung für abgehende und eingehende Schüler*innen birgt eine zielführende Vernetzung mit außerschulischen Akteuren*innen wertvolle Potenziale. Darüber hinaus beschäftigt sich diese Dimension mit Fragen der Außendarstellung (Öffentlichkeitsarbeit, Werbung etc.). Hierin liegen nicht nur Möglichkeiten über inklusive Angebote der eigenen Schule zu informieren und damit zu werben, auch eine interne Öffentlichkeitsarbeit lässt sich nutzen, beispielsweise zur Erarbeitung des Leitbildes oder einer gelingenden Kommunikation in der Schule.

Der Blick nach innen kommt in der Dimension des Schullebens zum Tragen und richtet sich auf Faktoren, die das Schulleben außerhalb des Kerngeschäfts Unterricht bestimmen und wieder alle Akteursebenen betreffen (können). Insbesondere Schüler*innen haben hier die Möglichkeit zur aktiven Beteiligung an Schulentwicklungsprozessen. Dies sind vordergründig Maßnahmen zur Gestaltung des außerunterrichtlichen Schulalltags (Projekttage, GTA Angebote usw.), die Beziehungsgestaltung zwischen den schulischen Akteuren*innen über den Unterricht hinaus, sowie - ganz zentral - die Etablierung eines inklusiven Schulklimas. Die aktive Gestaltung des Schullebens obliegt sämtlichen beteiligten Akteuren*innen und ermöglicht über unterschiedliche Formen der Zusammenarbeit die Schaffung von Gemeinschaftserfahrungen. Insofern kommt der Förderung von Formen der Zusammenarbeit - auch über den Unterricht hinaus - eine wichtige Rolle für die Schaffung einer inklusiven Schule zu; nicht zuletzt da Zusammenarbeit als 
»Grundwert einer inklusiven Schulkultur « (Urton et al. 2018, 42) verstanden werden kann. Unterschieden werden können dabei Beziehungen zwischen den Schüler*innen untereinander, zwischen schulischem Personal und Schüler*innen, den Lehrenden untereinander und mit der Schulleitung wie auch die Zusammenarbeit mit den Eltern. Geteilte Einsichten und Überzeugungen der einzelnen schulischen Mitglieder sowie die gemeinsame Arbeit an vorab formulierten Zielen können zum Ausgangspunkt der kontinuierlichen Etablierung und Gestaltung eines inklusiven Schulklimas genommen werden. Schule soll, darauf verweisen auch die vorliegenden Untersuchungsergebnisse, nicht nur ein Ort der Erziehung und Bildung sein. Opp (1997) spricht von Schule als Caring-Community und verweist darauf, dass Schule einen Ort der Beziehungen darstellen soll, der für alle einen positiven Erfahrungsraum bietet und Zugehörigkeit vermittelt. Dazu gehören auch die gelebte Antidiskriminierung und Antirassismus zur Vermeidung von Ausgrenzungen sowie die »Reduktion kategorialer, identitärer Zuschreibungen « (Gomolla und Radtke 2009). In 46,5\% der Fälle berichteten befragte Schüler*innen der untersuchten Schulen von Mobbingerfahrungen (selbst oder in anderen Fällen) sowie in $40,4 \%$ vom Erleben körperlicher Gewalt. Dies sind alarmierende Ergebnisse, die auf die Notwendigkeit weiterer Maßnahmen zur Schaffung eines positiven Schulklimas sowie einer positiven Willkommenskultur verweisen. Im Rahmen konkreter außerunterrichtlicher Maßnahmen, wie zum Beispiel die Initiierung von Projekten, Exkursionen etc. oder auch symbolischer Artefakte (Schullogo, symbolische Schirmherrschaften etc.) oder Rituale (z. B. die Etablierung einer spezifischen Willkommenskultur) können inklusive Bestrebungen gezielt zum Thema gemacht werden.

Wie oben bereits erwähnt, verfügt der Großteil der Pilotschulen über eine moderne Ausstattung für die Gestaltung von Unterrichtsprozessen und den gemeinsamen innerschulischen Austausch (interaktive Tafeln, PC-Kabinette, organisationsinterne digitale Kommunikations- und Organisationsplattformen). Insbesondere in Bezug auf die Nutzung dieser digitalen Ressourcen werden allerdings neue Herausforderungen offenbar. Als eigenständige Dimension hat daher das Thema Digitalisierung Einzug in das Gestaltungsmodell gehalten. Die aktuelle Brisanz der Thematik (Stichwort »Digitalpakt «) spiegelt sich auch in den Ergebnissen der vorliegenden Daten und fokussiert auf die jeweils vorhandene Ausstattung sowie den Einsatz neuer Medien und Technologien für unterrichtliche und außerunterrichtliche Belange: »Unbestritten ist, dass digitale Technologien, Anwendungen und Programme Wege für einen flexiblen, zeit- und ortsunabhängigen Bildungserwerb eröffnen. Sie können individualisiertes und kooperatives Lernen erleichtern und helfen, Inklusion zu verwirklichen. Angesicht der oft wenig erprobten Vielfalt an Angeboten und Möglichkeiten bedürfen sie aber einer intensiven Begleitung und Evaluierung « (BMBF 2016, 3). Zur dafür nötigen Ausstattung zählen unter anderem Schulnetzte, ein breitbandiger Internetzugang, moderne Hardware und Software sowie internetbasierte Dienste und Lernplattformen (Breiter, Zeising und Stolpmann 2015), für deren inklusi- 
ons-förderlichen Einsatz zusätzlich technischer Support und pädagogische Konzepte. Die Potenziale neuer Technologien liegen zum einen auf der Ebene der Unterrichtsgestaltung, indem sie beispielsweise niederschwellige und bedarfsorientierte Lernunterstützung bieten können und den Umgang mit Heterogenität maßgeblich erleichtern (Vaupel 2012). Auf der anderen Seite ermöglichen sie neue Formen der Kommunikation, Organisation und Zusammenarbeit. Voraussetzung hierfür sind sowohl Akzeptanz aufseiten der Nutzer*innen wie auch entsprechende medienpädagogische und -didaktische Kompetenzen.

Alle von den Schulen identifizierten Anliegen werden eingebettet in einen, alle Handlungs- und Steuerdimensionen umfassenden Schulentwicklungsprozess. Alle anstehenden Prozesse im Sinne einer perspektivischen Entwicklungsplanung und Zukunftsgestaltung werden hierüber aufgezeigt. Gelingende Schulentwicklung wird im Projekt verstanden als systematischer, zielgerichteter Prozess der strategischen Ressourcenauswahl (personell, materiell, finanziell) und deren Verknüpfung zu erfolgreichen Handlungen, um spezifisch situative und komplexe Herausforderungen nachhaltig zu lösen (Schreyögg und Eberl 2015) und die Schule als Organisation, ihre Akteure*innen und den Unterricht weiterzuentwickeln. Alle, in den einzelnen Feldern und Ebenen erfolgten Bearbeitungsschritte laufen im Schulentwicklungsprozess zusammen: Welche konkreten Ziele (normativ, strategisch und operativ) werden verfolgt, wie werden sie umgesetzt, gesteuert und evaluiert, wer trägt Entscheidungen, welche Instrumente der Dokumentation und des Wissensmanagements wurden ausgewählt, welche Kriterien des Qualitätsmanagements definiert und Rollen und Verantwortlichkeiten zugewiesen. Im Sinne einer transparenten Arbeitsgrundlage für eine systematische Schulentwicklung und zur kontinuierlichen Überprüfung des Erreichten werden sämtliche Schritte - bestenfalls - in einem Schulprogramm festgehalten (Landesamt für Schule und Bildung 2016, 7f.). Es empfiehlt sich, das Schulprogramm zum Ausgangspunkt für sämtliche Schulentwicklungsbestrebungen zu erklären und unter der Maßgabe prozessbegleitender Konsensbildung als strategisches Instrument für die Schulentwicklung zu nutzen. Mit der schriftlich fixierten Form eines Schulprogramms »erlangt es für alle schulischen Akteure[ ${ }^{*}$ innen] die notwendige Konkretisierung, Transparenz und Verbindlichkeit « (ebd.).

Gleichermaßen unterliegen Schulentwicklungsprozesse auch immer konstitutiven, bildungspolitischen, institutionellen, gesamtgesellschaftlichen und finanziellen Rahmenbedingungen bzw. Kontextvariablen (siehe Abschnitt Anforderungen). Sie markieren die Grenzen des schulspezifischen Aktionsraumes und sind nur eingeschränkt beeinflussbar. Abgesehen von dem »verpflichtenden Aufbau eines inklusiven Bildungssystems (Hennemann et al.2018,4), zeigt sich bezogen aufdie Umsetzung von Inklusion, dass es » bedingt durch die föderale Bildungspolitik [...] keine einheitliche Umsetzung von Inklusion gibt « (Urton et al. 2018, 46). Die Wahl der beteiligten Schulen auf diejenigen mit Standort in Sachsen zu beschränken, hatte vor diesem Hintergrund den Vorteil des landesspezifischen Geltungsbereichs der sächsischen Landesbildungspolitik. 


\section{Ausblick}

Der Artikel stellt erste Einblicke in die laufende Projektarbeit bereit, indem die auf Basis einer theoriebasierten Exploration (Bortz und Döring 2000) eruierten Dimensionen inklusiver Handlungsfelder an Schulen skizziert wurden. Diese werden im Rahmen eines groß angelegten Mixed-Methods-Designs an fünf verschiedenen Pilotschulen wissenschaftlich evaluiert und pilotiert. Die Studie ermöglicht damit eine evidenzbasierte Entwicklung eines formalen Gestaltungsmodells für inklusive Schulentwicklungsprozesse und liefert einen zentralen Beitrag zu theoretisch und empirisch fundierten Gelingensbedingungen für Inklusion im schulischen Kontext. In einem nächsten Schritt wird der aktuelle Arbeitsstand, wie es hier skizziert wurde, sowohl um die gewonnen empirischen Daten der Einzelschulen erweitert als auch um die jeweiligen Wechselwirkungen der einzelnen Dimensionen und Handlungsfelder angereichert. Die theoretisch ableitbaren Zusammenhänge, wie beispielsweise der zwischen inklusionsbezogenen Überzeugungen und der Wirksamkeit eines inklusiven Unterrichts (Jordan et al. 1993), gehören zu den zentralen Untersuchungsgegenständen der beschriebenen Ist-Stand-Erhebung und fließen als Ergebnisse in das Modell in Form von Wechselwirkungsmarkierungen ein, wie sie bislang nur (als gestrichelte Verbindungslinien) angedeutet wurden. Nach der Pilotierung des Gestaltungsmodells an den Schulen und einer zeitversetzten Abschlussevaluation wird es zum Ende des Projektes möglich sein, diejenigen Gelingensbedingungen, die hier auf theoretischer Basis nur angedeutet werden konnten, als tatsächlich funktional auszuweisen. Zentral für die erfolgreiche Implementierung inklusiver schulischer Strukturen erscheinen dabei vor allem zwei Aspekte:

Zum einen ist das Projekt Organisationsentwicklung inklusiver Schulen als Mehrebenenanalyse angelegt. Dies meint sowohl die Einbeziehung aller innerschulischer Ebenen (Schüler*innen, Lehrkräfte, Schulleitung) als auch außerschulische Instanzen (Schule, Schulträger, Eltern) unter Berücksichtigung gesellschaftlicher Normen und Werte und dem damit verbundenen staatlichen Bildungsauftrag. Spiess (1997) betont in diesem Zusammenhang, dass gute schulische Qualität immer dann angenommen werden kann, wenn es gelingt, Interessen und Bedürfnisse aller am Prozess Beteiligten zu berücksichtigen. »Gesteuerte Ideen « (I1, Z 400) und »Visionen der Schulleitung « (I1, Z 409) laufen Gefahr, kollektive Überzeugungen zu spalten und damit den Zugang zur Handlungspraxis zu verwehren. Eine Verknappung der Perspektiven auf die scheinbar primären Handlungsakteure wie Schulleitung oder auch Steuergruppen führt zwangsläufig zu Partizipationsverlusten und letztendlich zum Verlust der Quellen zur Gestaltung sozialer Institutionen (Fend 1996).

Zum anderen meint Schule als lernende Organisation nicht, dass diese sich Entwicklungsherausforderungen, wie die der Schaffung inklusiver Strukturen, ohne Unterstüt- 
zung und auch Kontrollmöglichkeiten von außen stellen muss. Eine Prozessbegleitung, so wie es das Projekt vorsieht, eröffnet einen von außen und wissenschaftlich fundierten Reflexionsraum, um die eigene pädagogische Praxis zukunftsweisend auf den Prüfstand $\mathrm{zu}$ stellen. Ziel ist es letztendlich, ein innerschulisches Management zu etablieren, das durch Transparenz, Mitbestimmung und Verantwortungsteilung geprägt ist, um das gesamte Schulgeschehen und die damit verbundenen Aufgaben und Herausforderungen eigenständig zu erkennen, zu reflektieren sowie systematisch und nachhaltig daran zu arbeiten.

\section{Anmerkungen}

1 Das Forschungsprojekt »Organisationsentwicklung inklusiver Schulen« ist ein Teilprojekt des Verbundprojektes "Schule inklusiv gestalten (SING) - Entwicklung fachdidaktischer Konzepte und organisatorischer Strukturen einer inklusiven Schule« gemeinsam mit der TU Dresden und der Bergischen Universität Wuppertal. Das Verbundprojekt wird im Rahmen der Förderrichtlinie des BMBFs »Qualifizierung pädagogischer Fachkräfte für inklusive Bildung (Förderprogramm »Empirische Bildungsforschung«) gefördert. Das Teilprojekt ist am Fraunhofer Institut für Internationales Management und Wissensökonomie (IMW) in Leipzig angesiedelt (Teilprojektleitung Anzhela Preissler).

2 Weiterführende Informationen: https://www.empirische-bildungsforschung-bmbf.de/de/ 2292.php

3 Weiterführende Informationen:https://www.imw.fraunhofer.de/de/abteilungen-und-gruppen/ unternehmensentwicklung/qualifizierungs-und-kompetenzmanagement/projekte/SING.html

4 Im Beschluss der KMK von 2004 wurde unter anderem die Differenzierung, Integration und Förderung von Diversität und Heterogenität als Kompetenzbereich der Lehrerbildung festgehalten, in den ländergemeinsamen inhaltlichen Anforderungen für die Fachwissenschaften und Fachdidaktiken hielt in den fachspezifischen Kompetenzprofilen die unterrichtliche Gestaltung unter Berücksichtigung von Inklusion Einzug.

5 In Sachsen - dem Bundesland der im Projekt untersuchten Schulen - gibt es, anders als in anderen Bundesländern, beispielsweise Bremen, Schleswig-Holstein), noch Förderschulen, wobei Kinder mit einem Förderbedarf sowohl an einer Regel- als auch an einer Förderschule unterrichtet werden können.

6 Im Projekt SING sind es sogar 73,2\% des befragten schulischen Personals, die eine gemeinsame Unterrichtung von Kindern mit und ohne sonderpädagogischen Förderbedarf für sinnvoll halten.

7 die Autorinnen nutzen den Begriff der Integration im Zitat mit Bezug auf ältere Studien, ansonsten verwenden sie den Inklusionsbegriff

8 Die Dimensionen der Personalentwicklung, in deren Mittelpunkt die Professionalisierung der Lehrenden liegen, sowie die Unterrichtsentwicklung mit der Beachtung didaktischer Konzepte, werden im unten skizzierten Modell als zwei der fünf Handlungsfelder mitgedacht.

9 In der Zusammenarbeit mit den schulischen Akteuren*innen wird für das entstandene Gestaltungsmodell nicht der Begriff der Balanced Scorecard, sondern der der "Strategischen Landkarte« verwendet.

10 Der Blick auf Modellprojekte und Praxisberichte im Umgang mit Balanced Scorecards sind marginal, jedoch vereinzelt schulformspezifisch vorzufinden. Im Rahmen der Stiftung Bil- 
dungspaket Bayern wurde an einer Haupt-, Wirtschaftsschule und einem Gymnasium das Schulmanagement-Programm KOLLUX eingeführt und erprobt.

11 Erste Analysen der Interviews mit Schulleitungen und dem schulischen Personal verweisen bereits jetzt auf eine Bandbreite an unterschiedlichen Begriffsverständnissen von Inklusion wie auch Prioritätensetzungen bezüglich zu initiierender Umsetzungsstrategien und Instrumente.

12 Da es sich um ein bis 12/2020 laufendes Projekt im Bereich der anwendungsorientierten Forschung handelt, ist aufgrund des kontinuierlichen Austauschs mit der Nutzergruppe mit einer iterativen Weiterentwicklung des Modells zu rechnen.

13 Auf die Frage, inwieweit neben der Kernaufgabe des Unterrichtens weitere Funktionen wahrgenommen werden, berichtet eine Pädagogin von vielfältigen, kaum mehr zu vereinbarenden Erfordernissen, die zu bewältig sind: Neben der Teilnahme an Teamsitzungen und der Mitwirkung in diversen Arbeitskreisen liegt ein Schwerpunkt der Arbeit auf individuellen Betreuungs- und Beratungsleistungen für Schüler*innen, für die nach Aussage der Interviewpartnerin allein 5-6 Stunden pro Woche aufgewendet werden. Hinzu kommen dafür nötige Abstimmungs- und Kommunikationsprozesse mit Kollegen*innen, die zum Teil »nur noch zwischen Tür und Angel« abgehalten werden. Insgesamt wird dieser Zustand als hohe kollektive Belastung im schulischen Alltag bewertet: »Es ist zu viel, es belastet uns, wir kommen eigentlich... wir werden den Aufgaben nicht mehr gerecht« (I1, Z. 511-513).

14 Berufsbezogene Überzeugungen werden teilweise synonym mit verschiedenen Begriffen, wie Haltungen, Einstellungen und auch beliefs verwendet. Wir nutzen den Begriff der Überzeugung und orientieren uns dabei an der Definition von Reusser und Pauli (2014), welche „Vorstellungen über das Wesen und die Natur von Lehr-Lernprozessen, Lerninhalten, die Identität und Rolle von Lernenden und Lehrenden (sich selbst) sowie den institutionellen und gesellschaftlichen Kontext von Bildung und Erziehung " umschreiben und letztendlich »berufsbezogenen Denken und Handeln Struktur, Halt, Sicherheit und Orientierung geben« (642).

\section{Literatur}

Amrhein, Bettina. 2011. Inklusion in der Sekundarstufe. Eine empirische Analyse. Bad Heilbrunn: Julius Klinkhardt.

Amrhein, Bettina und Myrle Dziak-Mahler. 2014. Fachdidaktik inklusiv: Auf der Suche nach didaktischen Leitlinien für den Umgang mit Vielfalt in der Schule. Münster: Waxmann.

Artiles, Alfredo J. und Alan Dyson. 2005. Inclusive education in the globalization age: The promise of comparative cultural historical analysis, herausgegeben von Mitchell David, 37-62. London: Routledge.

Beauftragte der Bundesregierung für die Belange von Menschen mit Behinderungen. 2017. Die UNBehindertenrechtskonvention: Übereinkommen über die Rechte von Menschen mit Behinderungen. Berlin: Beauftragte der Bundesregierung für die Belange von Menschen mit Behinderungen. https://www.behindertenbeauftragte.de/SharedDocs/Publikationen/UN_Konvention_ deutsch.pdf?_blob=publicationFile\&v= 2 (Stand 08.04.2019).

Berglehner, Florian und Karl Wilbers. 2015. "Schulisches Prozessmanagement - eine Einführung." In Schulisches Prozessmanagement Einführung, Praxisreflexion, Perspektiven, herausgegeben von Florian Berglehner und Karl Wilbers, 17-91. Berlin: epubli.

Berkemeyer, Nils. 2010. Die Steuerung des Schulsystems: Theoretische und praktische Explorationen. Wiesbaden: VS Verlag für Sozialwissenschaften. 
Boer, Heike de und Sabine Reh. 2012. Beobachtung in der Schule - Beobachtungen lernen. Wiesbaden: VS Verlag für Sozialwissenschaften.

Bortz, Jürgen und Nicola Döring. 2000. Forschungsmethoden und Evaluation für Sozialwissenschaftler. Heidelberg: Springer-Medizin-Verlag.

Bosse, Ingo. 2017. „Gestaltungsprinzipien für digitale Lernmittel im Gemeinsamen Unterricht. Eine explorative Studie am Beispiel der Lernplattform Planet Schule.«In Jahrbuch Medienpädagogik 13, herausgegeben von Kerstin Mayrberger, Johannes Fromme, Petra Grell und Theo Hig, 151-164. Wiesbaden: Springer VS.

Bosse, Ingo. 2018. "Qualitätskriterien für audiovisuelle und digitale Medien für den inklusiven Unterricht -eine Evaluationsstudie des digitalen Angebots `Planet Schule«. Zeitschrift für Heilpädagogik 69 (6): 256-270.

Braches-Chyrek, Rita. 2015. Neue Disziplinäre Ansätze in der Sozialen Arbeit: Eine Einführung. Leverkusen-Opladen: Barbara Budrich-Esser.

Breiter, Andreas, Anja Zeising und Björn E. Stolpmann. 2015. Szenarien lernförderlicher IT-Infrastrukturen in Schulen. Betriebskonzepte, Ressourcenbedarf und Handlungsempfehlungen. Gütersloh: Bertelsmann Stiftung. https://www.bertelsmann-stiftung.de/fileadmin/files/BSt/ Publikationen/GrauePublikationen/Studie_IB_IT_Infrastruktur_2015.pdf

Boban, Ines und Andreas Hinz. 2003. Index für Inklusion. Lernen und Teilhabe in der Schule der Vielfalt entwickeln (deutsche Übersetzung). Halle (Saale): Martin-Luther-Universität Halle-Wittenberg. https://www.eenet.org.uk/resources/docs/Index\%20German.pdf

Bundesministerium für Bildung und Forschung. 2016. Bildungsoffensive für die digitale Wissensgesellschaft. Berlin: BMBF. https://www.bmbf.de/files/Bildungsoffensive_fuer_die_digitale_ Wissensgesellschaft.pdf

Deutscher Gewerkschaftsbund. 2015. Gemeinsam statt getrennt - gewerkschaftliche Kriterien für die Entwicklung inklusiver Schulen. Berlin: DGB-Bundesvorstand. https://www.gew.de/aktuelles/ detailseite/neuigkeiten/dgb-forderungen-fuer-eine-gute-inklusive-schule/ (12.04.2019).

Deutsche Unesco-Kommission. 2014. Inklusion. Leitlinien für die Bildungspolitik, herausgegeben von Katja Römer. Bonn: Dt. UNESCO-Kommission e.V.

Dubs, Rolf. 2006. »Führung. «In Professionswissen Schulleitung, herausgegeben von Herbert Buchen und Hans-Günter Rolff, 102-176. Weinheim: Beltz.

Egger, Marina. 2017. „Der Qualitätsdiskurs im Bildungssystem und der Inklusionsanspruch.« In Inklusion und Schulentwicklung, herausgegeben von Vera Moser und Marina Egger, 31-55. Stuttgart: Kohlhammer.

Fegert, Jörg M. und Renate Schepker. 2014. »Ambivalenzen um die Inklusion. « Zeitschrift für Kinderund Jugendpsychiatrie 42(5): 297-299. doi: 10.1024/1422-4917/a000305

Fend, Helmut. 1996. »Schulkultur und Schulqualität. «In Die Institutionalisierung von Lehren und Lernen. Beiträge zu einer Theorie der Schule, Zeitschrift für Pädagogik, Beiheft 34, herausgegeben von Achim Leschinsky, 85-97. Weinheim et al.: Beltz 1996.

Fend, Helmut. 2008. Dimensionen von Qualität im Bildungswesen: Von Produktindikatoren zu Prozessindikatoren am Beispiel der Schule. Weinheim u.a: Beltz.

Fischer, Christian, Henrike Kopmann, David Rott, Marcel Veber und Horst Zeinz. 2014. »Adaptive Lehrkompetenz und pädagogische Haltung." In Jahrbuch für allgemeine Didaktik 2014. Thementeil Allgemeine Didaktik für eine inklusive Schule. Allgemeiner Teil, herausgegeben von Klaus Zierer, 16-34. Hohengehren: Schneider Verlag.

Frühauf, Theo. 2008. „Von der Integration zur Inklusion - ein Überblick.« In Von der Integration zur Inklusion. Grundlagen - Perspektiven - Praxis., herausgegeben von Andreas Hinz, Ingrid Körner und Ulrich Niehoff, 11-32. Marburg: Lebenshilfe.

Forsa. 2017. Inklusion an Schulen aus Sicht der Lehrkräfte in Deutschland - Meinungen, Einstel- 
lungen, Erfahrungen. Ergebnisse einer repräsentativen Lehrerbefragung. Berlin: forsa Politik- und Sozialforschung GmbH. https://www.vbe.de/fileadmin/user_upload/VBE/Service/ Meinungsumfragen/2017_05_10_Inklusion_an_Schulen_Auswertung.pdf

Füssel, Marian. 2007. Michel de Certeau: Geschichte, Kultur, Religion. Konstanz: UVK Verlagsgesellschaft $\mathrm{mbH}$.

Gomolla, Mechtild und Frank-Olaf Radtke. 2009. Institutionelle Diskriminierung: Die Herstellung ethischer Differenz in der Schule. Wiesbaden: Springer VS.

Görtler, Sophie-Cathérine. 2017. »Instrumente der inklusiven Schulentwicklung.« In Inklusion und Schulentwicklung: Konzepte, Instrumente, Befunde, herausgegeben von Vera Moser und Marina Egger, 144-161. Stuttgart: Kohlhammer.

Heinrich, Martin, Michael Urban und Rolf Werning. 2013. »Grundlagen, Handlungsstrategien und Forschungsperspektiven für die Ausbildung und Professionalisierung von Fachkräften für inklusive Schule. «In Inklusive Bildung professionell gestalten. Situationsanalyse und Handlungsempfehlungen, herausgegeben von Hans Döbert und Horst Weishaupt, 69-133. Münster et al.: Waxmann.

Hennemann, Thomas, Jürgen Wilbert und Clemens Hillenbrand. 2014. Wissenschaftliche Begleitung im Rahmen der Umsetzung zur inklusiven Schule im Kreis Mettmann: Mehrebenenanalyse 2010-2012. Abschlussbericht. Köln: Lehrstuhl für Erziehungshilfe und sozial-emotionale Entwicklungsförderung Universität zu Köln.

Hess, Doris, Michael Ruland, Maurice Meyer und Jacob Steinwede. 2019. Schulische Inklusion. Untersuchung zu Einstellungen zu schulischer Inklusion und Wirkungen im Bildungsverlauf. Bonn: infas Institut für angewandte Sozialwissenschaft GmbH. https://www.aktion-mensch. de/inklusion/dam/jcr:b64adbd9-4a2b-4a79-b3ac-98591937293f/Studie\%20inkl.Bildung_ Langfassung_barrierefrei.pdf

Jerusalem, Matthias (Hrsg.) und Dieter Hopf (Hrsg.). 2002. Selbstwirksamkeit und Motivationsprozesse in Bildungsinstitutionen. Weinheim und Basel: Beltz, S. 28-53. - (Zeitschrift für Pädagogik, Beiheft; 44).

Jordan, Anne, Gonul Kircaali-Iftar und C. T. Patrick Diamond. 1993. „Who has a Problem, the Student or the Teacher? Differences in Teachers' Beliefs. About Their Work with At-risk and Integrated Exceptional Students."International Journal of Disability, Development and Education 40, no 1: 45-62. doi: 10.1080/0156655930400104

Kaplan, Robert S. 1997. Balanced Scorecard: Strategien erfolgreich umsetzen. Stuttgart: Schäffer-Poeschel (Handelsblatt-Reihe).

Kaplan, Robert S. und David P. Norton. 2004. Strategy Maps: Converting Intangible Assets into Tangible Outcomes. Boston: Harvard Business Review Press.

KOLLUX. 2008. Leitfaden für das Schulmanagement-Programm 2008. Berlin: Cornelsen.

Kultusministerkonferenz. 2004. Standards für die Lehrerbildung: Bildungswissenschaften. Beschluss der Kultusministerkonferenz vom 16.12.2004. Berlin: Sekretariat der Kultusministerkonferenz. https://www.kmk.org/fileadmin/veroeffentlichungen_beschluesse/2004/2004_12_16

-Standards-Lehrerbildung.pdf (08.04.2019).

Kultusministerkonferenz. 2018. Ländergemeinsame inhaltliche Anforderungen für die Fachwissenschaften und Fachdidaktiken in der Lehrerbildung: Beschluss der Kulturministerkonferenz vom 16.10.2008i. d. F.vom 11.10.2018. Berlin: Sekretariat der Kultusministerkonferenz. https://www. kmk.org/fileadmin/veroeffentlichungen_beschluesse/2008/2008_10_16-Fachprofile -Lehrerbildung.pdf (08.04.2019).

Kunter, Mareike und Britta Pohlmann. 2009. »Lehrer.« In Pädagogische Psychologie, herausgegeben von Elke Wild und Jens Möller, 262-280. Heidelberg: Springer Verlag.

Landesamt für Schule und Bildung (Hrsg.). 2016. Schulprogrammarbeit an sächsischen Schulen. Ra- 
debeul: Sächsisches Bildungsinstitut. https://publikationen.sachsen.de/bdb/artikel/26707/ documents/38681

Langenohl, Andreas. 2008. „Die Schule als Organisation. Gefälligkeitsübersetzung: The school as an organization.". In Lehr(er)buch Soziologie. Für die pädagogischen und soziologischen Studiengänge, herausgegeben von Herbert Willems, 817-833. Wiesbaden: VS Verlag für Sozialwissenschaften.

Locke, Edwin A. 1976. »The nature and causes of job satisfaction. «In Handbook of industrial and organizational psychology, herausgegeben von Marvin D. Dunnette, 1297-1349. Chicago: Rand McNally.

Mayring, Philipp. 2008. Qualitative Inhaltsanalyse: Grundlagen und Techniken. Weinheim: Beltz.

Melzer, Conny, Clemens Hillenbrand, David Sprenger und Thomas Hennemann. 2015. »Aufgaben von Lehrkräften in inklusiven Bildungssystemen - Review internationaler Studien.« Erziehungswissenschaft 26 (51): 61-80.

Moser, Vera. 2017. »Inklusion und Organisationsentwicklung." In Inklusion und Schulentwicklung, herausgegeben von Vera Moser und Marina Egger, 15-30. Stuttgart: Kohlhammer.

Moser, Vera und Marina Egger. 2017. »Einleitung." In Inklusion und Schulentwicklung, herausgegeben von Vera Moser und Marina Egger, 9-14. Stuttgart: Kohlhammer.

Opp, Günther. 1997. »Die Schule als `Caring-Community.« In Geschlechterverhältnisse in der Behindertenpädagogik: Subjekt/Objekt-Verhältnisse in Wissenschaft und Praxis, herausgegeben von Wolfang Jantzen, 146-154. Luzern: Edition SZH/CSPS.

Rolff, Hans-Günter. 2007. Studien zu einer Theorie der Schulentwicklung. Weinheim: Belz.

Rolff, Hans-Günter. 2016. Schulentwicklung kompakt: Modelle, Instrumente, Perspektiven. Weinheim: Beltz.

Pitts Santoli, Susan, John Sachs, Elizabeth A. Romey und Stephen McClurg. 2008. »A Successful Formula for Middle School Inclusion: Collaboration, Time and Administrative Support.« RMLE Online 32, no 2: 1-13. https://doi.org/10.1080/19404476.2008.11462055.

Scheer, David und Désirée Laubenstein. 2018. Schulische Inklusion entwickeln. Stuttgart: Kohlhammer.

Schwab, Susanne. (2015). »Einflussfaktoren auf die Einstellung von Schülerlnnen gegenüber Peers mit unterschiedlichen Behinderungen."Zeitschrift für Entwicklungspsychologie und Pädagogische Psychologie 47 (4): 177-187.

Soodak, Leslie C. 2003. »Classroom Management in Inclusive Settings. « Theory into Practice 42, no.4 (Herbst): 327-333. doi: 10.1207/s15430421 tip4204_10

Spiess, Kurt. 1997. Qualität und Qualitätsentwicklung. Eine Einführung. Arau: Sauerländer.

The Boston Consulting Group. 1988. Vision und Strategie. München: Econ.

Trumpa, Silke, Frauke Janz, Vera Heyl und Stefanie Seifried. 2014. »Einstellung zu Inklusion bei Lehrkräften und Eltern - Eine schulartspezifische Analyse.» Zeitschrift für Bildungsforschung 4, no. 3 (November): 241-256.

Heyl, Vera und Stefanie Seifried. 2014. »Inklusion? Da ist ja sowieso jeder dafür!? « Einstellungsforschung zu Inklusion." In Inklusive Bildung. Erkenntnisse und Konzepte aus Fachdidaktik und Sonderpädagogik, herausgegeben von Silke Trumpa, Stefanie Seifried, Eva-Kristina Franz und Theo Klauß, 47-60. Weinheim et al.: Beltz Juventa.

UNESCO. 1996. Die Salamanca Erklärung und der Aktionsrahmen zur Pädagogik für besondere Bedürfnisse angenommen von der Weltkonferenz »Pädagogik für besondere Bedürfnisse: Zugang und Qualität.«Spanien, 7. - 10. Juni 1994. Wien: Österreichische UNESCO Kommission.

Urton, Karolina, Moritz Börnert-Ringleb, Johanna Krull, Jürgen Wilbert und Thomas Hennemann. 2018. »Inklusives Schulklima: Konzeptionelle Darstellung eines Rahmen-modells.« Zeitschrift für Heilpädagogik 69: 40-52. 
Vaupel, Wolfgang. 2012. »Medienkompetenz ist Lernkompetenz." In Informationskompetenz und inklusive Mediengesellschaft: Dokumentation einer Fachtagung mit Projektbeispielen, herausgegeben von Harald Gapski, 25-28. Düsseldorf: kopaed-Verlag.

Wagner, Cornelia und Jana Rückmann. 2017. »Qualitätsmanagement in der beruflichen Bildung in Deutschland: Ein aktueller Überblick mit dem Schwerpunkt der schulinternen Evaluation. «In Berufsbildung, eine Renaissance?: Motor für Innovation, Beschäftigung, Teilhabe, Aufstieg, Wohlstand, ..., herausgegeben von Peter Schlögl, Michaela Stock, Daniela Moser, Kurt Schmid und Franz Gramlinger, 253-264. Bielefeld: W. Bertelsmann Verlag.

Wember, Franz B. 2013. »Herausforderung Inklusion: Ein präventiv orientiertes Modell schulischen Lernens und vier zentrale Bedingungen inklusiver Unterrichtsentwicklung.« Zeitschrift für Heilpädagogik 10: 380-388.

Wilbers, Karl. 2012. Wirtschaftsunterricht gestalten: Eine traditionelle und handlungsorientierte Didaktik für kaufmännische Bildungsgänge. Berlin: epubli.

Wocken, Hans. (2011): „Über die Entkernung der Behindertenrechtskonvention Ein deutsches Trauerspiel in 14 Akten, mit einem Vorspiel und einem Abgesang.« Zeitschrift für Inklusion (4). https://www.inklusion-online.net/index.php/inklusion-online/article/view/80/80

UN-BRK 2008: Gesetz zu dem Übereinkommen der Vereinten Nationen vom 13. Dezember 2006 über die Rechte von Menschen mit Behinderungen sowie zu dem Fakultativprotokoll vom 13. Dezember 2006 zum Übereinkommen der Vereinten Nationen über die Rechte von Menschen mit Behinderungen. Bundesgesetzblatt Jahrgang 2008 Teil II Nr. 35, ausgegeben zu Bonn am 31. Dezember 2008. https://www.un.org/Depts/german/uebereinkommen/ ar61106-dbgbl.pdf (11.04.2019).

\section{Die Autorinnen}

Fanny Hösel ist wissenschaftliche Mitarbeiterin der Gruppe Qualifizierungs- und Kompetenzmanagement am Fraunhofer-Zentrum für Internationales Management und Wissensökonomie IMW. Ihre Arbeitsschwerpunkte sind: Erwachsenenbildung und Weiterbildung, Bildungs- und Lerntheorien, Biografieforschung und Qualitative Sozialforschung.

Kontakt: fanny.hoesel@imw.fraunhofer.de

Ariane Bößneck, Dr., ist Vertretungsprofessorin am Institut für Rehabilitationspädagogik an der Martin-Luther-Universität Halle-Wittenberg, Arbeitsbereich Gefühls- und Verhaltensstörungen. Ihre Arbeitsschwerpunkte sind: inklusive Schul- und Unterrichtsentwicklung, Präventions- und Transitionsforschung, Positive Peerkultur, Resilienzforschung sowie qualitative Forschungsmethoden und deren Triangulation.

Kontakt: ariane.boessneck@paedagogik.uni-halle.de

Anzhela Preissler ist Leiterin der Gruppe Qualifizierungs- und Kompetenzmanagement am Fraunhofer-Zentrum für Internationales Management und Wissensökonomie IMW. Ihre Arbeitsschwerpunkte sind: Kompetenzmanagement, Lernprozessmanagement und Organisationsentwicklung.

Kontakt: anzhela.preissler@imw.fraunhofer.de 